\title{
Torque ripple reduction in direct torque controlled five-phase induction motor using modified five-level torque comparator
}

\author{
YOGESH TATTE* and MOHAN AWARE \\ Department of Electrical Engineering, Visvesvaraya National Institute of Technology, Nagpur 440010, India \\ e-mail: ytatte@gmail.com
}

MS received 4 May 2016; revised 25 April 2017; accepted 3 June 2017; published online 7 February 2018

\begin{abstract}
The five-phase induction motor inherently has the minimal torque ripple. However, when it is controlled by direct torque control (DTC) technique, the torque ripple increases due to the presence of a hysteresis torque comparator. The classical five-level torque comparator is presented in the previous literatures to control the torque ripple. However, this comparator has the drawback of wrong selection of zero voltage vectors inside the inner band on the positive side of the comparator, which enables the torque ripple to increase and dc-link utilization to decrease. In this paper, in order to reduce the torque ripple and to increase the dc-link utilization, a modified five-level torque comparator is proposed, which selects either medium or small voltage vectors instead of zero voltage vectors inside the inner band on the positive side of the comparator. In addition to torque ripple reduction and improvement in dc-link utilization, the proposed comparator significantly improves the quality of phase current. All the available 32 voltage vectors are selected through the proposed five-level torque comparator based on the location of $x-y$ stator flux in order to eliminate the $x-y$ stator flux so as to obtain reduced distortion in the phase current. By employing all the available voltage vectors, the freedom of utilization of all voltage vectors in the five-phase induction motor DTC drive is availed. The proposed five-level torque comparator is compared to its classical five-level counterpart through simulation and experimental results in order to validate the proposed DTC strategy.
\end{abstract}

Keywords. Direct torque control; five-phase induction motor; torque ripple; dc-link utilization; $x-y$ stator current.

\section{Introduction}

The five-phase induction motor (IM) is introduced with its inherent advantages of reduced torque ripple, fault tolerance, reduction of current per phase, etc. [1-3]. Owing to these advantages, direct torque control (DTC) based on the classical hysteresis controller for three-phase IM [4] is extended for five-phase IM [5]. However, this DTC of five-phase IM based on the classical hysteresis controller [5] has the drawback of torque ripple, like in case of threephase DTC scheme, because of the involvement of hysteresis torque controller. Some modifications are presented in the classical structure of the DTC scheme to reduce the torque ripple in case of three-phase IM [6-9]. In case of five-phase IM, some developments are presented in the classical DTC scheme to improve its performance in the context of torque ripple reduction, $x-y$ stator flux elimination, improvement in dc-link utilization, improvement in speed response and reduction in demagnetization of stator flux during low-speed operation [10-12]. In [10], a sensorless DTC-controlled five-phase

*For correspondence
IM drive is proposed, wherein the voltage vectors are selected so that the $x-y$ stator flux is eliminated with full utilization of dc-bus voltage. In [11], the predictive torque control technique is introduced as an alternative to classical DTC, wherein the torque ripple minimization, $x-y$ component elimination and improvement in speed response are achieved. In [12], the performance of the five-phase IM in low-speed region is significantly improved using the DTC technique.

The classical three-level torque comparator is implemented in $[10,11]$ and the classical five-level torque comparator is implemented in [12] for designing the DTC method for five-phase IM. Since the classical five-level torque comparator has more bands compared with the classical three-level torque comparator, it reduces the torque ripple. However, the classical five-level torque comparator selects the zero voltage vectors inside the inner band on positive side, which enables the torque ripple to be increased and dc-link utilization to be reduced. By carefully selecting the voltage vectors inside the inner band on positive side of the five-level torque comparator, the reduction in torque ripple and improvement in dc-link utilization can be achieved. 
In this paper, the voltage vector selection through the classical five-level torque comparator is modified such that the zero voltage vectors inside the inner band on the positive side of the comparator are replaced either by medium or by small voltage vectors in order to reduce the torque ripple. The selection of voltage vectors through the proposed five-level torque comparator improves the dc-link utilization. In the proposed DTC technique the obtained stator current waveform is significantly improved compared with current waveform in the DTC technique with the classical torque comparator. This is the bonus advantage of using the proposed five-level torque comparator in the DTC scheme. The virtual voltage vectors of [12] are implemented in the proposed DTC scheme to eliminate the $x-y$ stator flux. In order to check the capability of proposed fivelevel torque comparator in reducing the torque ripple and improving the dc-link utilization, a comparative investigation with its classical counterpart [12] is carried out. Simulation and experimental results are presented to validate the proposed modified DTC strategy.

This paper is organized as follows. Section 2 discusses the modelling of five-phase IM. Section 3 focuses on the formation of virtual voltage vectors. Section 4 gives the design of proposed modified five-level torque comparator. Simulation and experimental results are provided in sections 5 and 6 , respectively. Section 7 deals with the conclusions from the presented work.

\section{Modelling of five-phase IM}

The $d-q$ stator flux $\left(\lambda_{d q}^{s}\right), d-q$ rotor flux $\left(\lambda_{d q}^{r}\right), x-y$ stator flux $\left(\lambda_{x y}^{s}\right)$ and $x-y$ rotor flux $\left(\lambda_{x y}^{r}\right)$ of the motor considering stationary reference frame can be written as [13]

$$
\begin{gathered}
\lambda_{d q}^{s}=\int\left(V_{d q}^{s}-i_{d q}^{s} r_{s}\right) d t \\
\lambda_{d q}^{r}=\int\left(V_{d q}^{r}-i_{d q}^{r} r_{r}\right) d t \\
\lambda_{x y}^{s}=\int\left(V_{x y}^{s}-i_{x y}^{s} r_{s}\right) d t \\
\lambda_{x y}^{r}=\int\left(V_{x y}^{r}-i_{x y}^{r} r_{r}\right) d t \\
\lambda_{d q}^{s}=\left(L_{l s}+L_{m}\right) i_{d q}^{s}+L_{m} i_{d q}^{r} \\
\lambda_{d q}^{r}=\left(L_{l r}+L_{m}\right) i_{d q}^{r}+L_{m} i_{d q}^{s} \\
\lambda_{x y}^{s}=L_{l s} i_{x y}^{s} \\
\lambda_{x y}^{r}=L_{l r} i_{x y}^{r}
\end{gathered}
$$

where $V_{d q}^{s}, V_{d q}^{r}, V_{x y}^{s}, V_{x y}^{r}$ represent, respectively, the stator and rotor voltage complex vectors representing $d-q$ and $x-y$ axis and $i_{d q}^{s}, i_{d q}^{r}, i_{x y}^{s}, i_{x y}^{r}$ represent the stator and rotor current complex vectors representing $d-q$ and $x-y$ axis; $r_{s}$ is stator resistance, $r_{r}$ is rotor resistance, $L_{l s}$ is the leakage stator inductance, $L_{l r}$ is the leakage rotor inductance and $L_{m}$ is the mutual inductance. The electromagnetic torque can be expressed as

$$
T_{e}=\frac{5}{2} P \frac{L_{m}}{\sigma L_{s} L_{r}} \operatorname{Im}\left[\lambda_{d q}^{s} \lambda_{d q}^{r *}\right]
$$

where $P$ is the number of pole pairs, ' $*$ ' denotes the complex conjugate, $L_{s}$ is the stator inductance, $L_{r}$ is the rotor inductance and leakage coefficient $(\sigma)=1-\frac{L_{m}^{2}}{L_{s} L_{r}}$. A schematic of five-phase IM drive is shown in figure 1a and in a balanced five-phase IM, the five stator phase groups are distributed with spacing of $72^{\circ}$ as shown in figure $1 \mathrm{~b}$. The five-phase inverter has $2^{5}=32$ switching voltage vectors consisting of 30 active and 2 zero. The switch function of these voltage vectors is represented as $S=\left[S_{A} S_{B} S_{C} S_{D}\right.$ $\left.S_{E}\right]^{\mathrm{T}}$, where $S_{i}=0$ or 1 , state 1 represents turning 'ON' of upper switch and for the same state, lower switch will be turned 'OFF'. State 0 represents turning 'ON' of lower switch and for the same state, upper switch will be turned 'OFF'.

\section{Formation of virtual voltage vectors group}

The $x-y$ space plane exists in the five-phase IM, which is decoupled and orthogonal to already existing $d-q$ space plane. The fundamental supply component and supply harmonics of the order $10 n \pm 1(n=0,1,2,3, \ldots)$ map into $d-q$ space plane and the supply harmonics of the order $10 n \pm 3(n=0,1,2,3, \ldots)$ map into $x-y$ space plane. The electromagnetic torque is produced by $d-q$ component only (Eq. (9c)) and $x-y$ component (Eq. (3)) simply causes losses in the machine by distorting the phase current. Hence, it becomes necessary to eliminate the $x-y$ stator flux while designing the DTC scheme for the five-phase IM. It is observed in figure $2 \mathrm{a}$ and $\mathrm{b}$ that the voltage vectors that have the same direction in $d-q$ space plane are located at different positions in $x-y$ space plane. For example, the voltage vectors $V_{1}, V_{11}$ and $V_{21}$ have the same direction in $d-q$ space plane as shown in figure $2 \mathrm{a}$. However, in $x-y$ space plane, the voltage vectors $V_{1}$ and $V_{21}$ are located in the same direction, but voltage vector $V_{11}$ is located in exactly opposite direction as shown in figure $2 \mathrm{~b}$. This peculiarity of location of voltage vectors in $d-q$ and $x-y$ space plane is used to eliminate the $x-y$ stator flux. In order to eliminate the $x-y$ stator flux, the virtual voltage vectors are formed. The large voltage vectors $V_{1}-V_{10}$ and medium voltage vectors $V_{11}-V_{20}$ are represented by a group of large virtual voltage vectors $V_{L X}$, where $X=1-10$. The medium voltage vectors $V_{11}-V_{20}$ and small voltage vectors $V_{21}-V_{30}$ are represented by a group of small virtual voltage vectors 


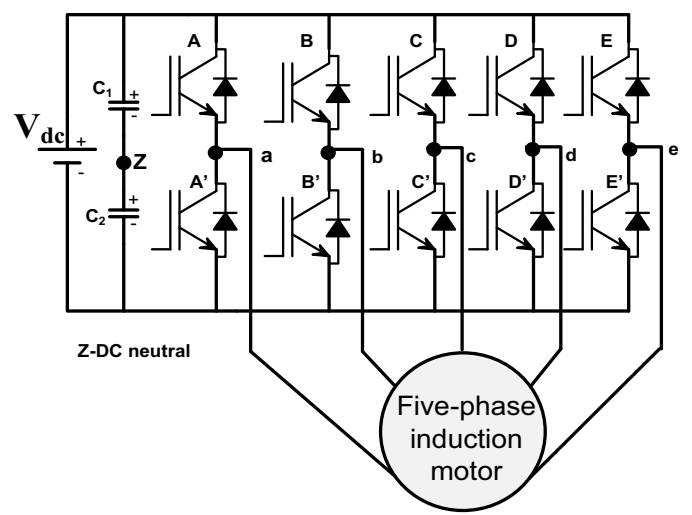

(a)

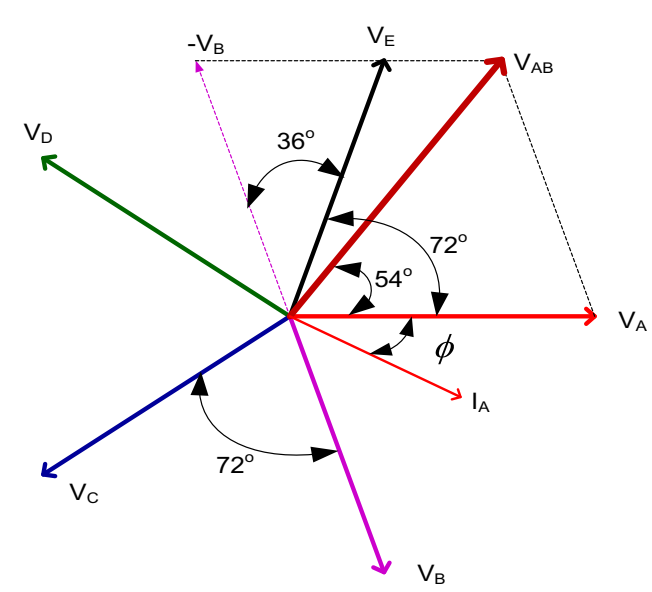

(b)

Figure 1. (a) Schematic of five-phase induction motor drive and (b) phasor diagram of five-phase system representing winding position.

$V_{S X}$, where $X=1-10$. The zero voltage vectors $V_{0}$ and $V_{31}$ remain as they are. These 20 virtual voltage vectors and two zero voltage vectors are divided into 10 sectors as shown in figure 2c [12]. Once $V_{L X}$ is selected by the control strategy, either large or medium voltage vector will be selected, depending upon the location of $x-y$ stator flux. For example, if large virtual voltage vector $V_{L 3}$ is selected and if $x-y$ stator flux is located in left half of $x-y$ space plane, the actual voltage vector $V_{3}$ will be selected as depicted in figure 2d, because $V_{3}$ is located opposite to left half of $x-y$ space plane. The selection of $V_{3}$ or $V_{13}$ will be done by the following procedure:

- check the location of $x-y$ stator flux $\left(\alpha=\tan ^{-1}\left(\lambda_{y}^{s} / \lambda_{x}^{s}\right)\right)$

- If $x-y$ stator flux lies in sector (V-IX), $V_{3}$ will be selected.

- If $x-y$ stator flux lies in sector (X-IV), $V_{13}$ will be selected.

Similarly, actual voltage vectors from the small virtual voltage vectors will be selected. The selection of opposite voltage vectors to the location of $x-y$ stator flux eliminates the $x-y$ stator flux [12].

\section{Implementation of proposed five-level torque comparator from classical five-level torque comparator}

In this section, firstly the formation of classical five-level torque comparator from classical three-level torque comparator is addressed; then the evolution of the proposed fivelevel torque comparator from the classical five-level torque comparator is focussed. It is already presented in the literatures $[9,12]$ that with increasing number of levels of torque comparator the torque ripple of machine can be reduced. The classical five-level torque comparator is therefore presented in $[9,12]$ to minimize the torque ripple compared with its threelevel counterpart. The bands on both sides of the classical three-level torque comparator $[10,11]$ as shown in figure $3 \mathrm{a}$ are divided into two parts in each side in order to convert the classical three-level torque comparator into classical five-level torque comparator [12] as shown in figure $3 b$. Out of five levels of the classical five-level torque comparator, outer two levels on both sides of the comparator, i.e., $H B \_2$ and $-H B \_2$ are used for increasing the torque and decreasing the torque, respectively, at fast rate, for which the large virtual voltage vectors are selected, inner two levels on both sides, i.e., $H B \_1$ and $-H B_{-} \_$are used for increasing the torque and decreasing the torque, respectively, at slow rate, for which the small virtual voltage vectors are selected, and the centre level is used when the torque error lies in between the inner two levels, for which the zero voltage vectors are selected. The outer bands (levels) guarantee the dynamic performance of the motor and the inner bands reduce the torque ripple.

The torque variation is expressed as [6-9]

$$
\begin{aligned}
\frac{d T_{e}}{d t}= & -T_{e 0}\left(\frac{r_{s}}{L_{s}}+\frac{r_{r}}{L_{r}}\right) \frac{1}{\sigma} \\
& +\frac{5}{2} P \frac{L_{m}}{\sigma L_{s} L_{r}} \operatorname{Im}\left[\left(V_{d q}^{s}-j \omega_{m} \lambda_{d q}^{s}\right) j \lambda_{d q}^{r}\right]
\end{aligned}
$$

where $T_{e 0}$ is the torque at current instant and $\omega_{m}$ is the motor angular velocity. It is investigated in [6-9] that the torque variation (Eq. (10)) is influenced by the length of applied voltage vector. The length of large, medium and small voltage vectors is, respectively, $0.647,0.4$ and $0.2472 V_{d c}$.

The virtual large voltage vectors consisting of large and medium voltage vectors have the dc-bus length of

$$
(0.647+0.4) V_{d c} / 2=0.5235 V_{d c} .
$$

The virtual small voltage vectors consisting of medium and small voltage vectors have the dc-bus length of 


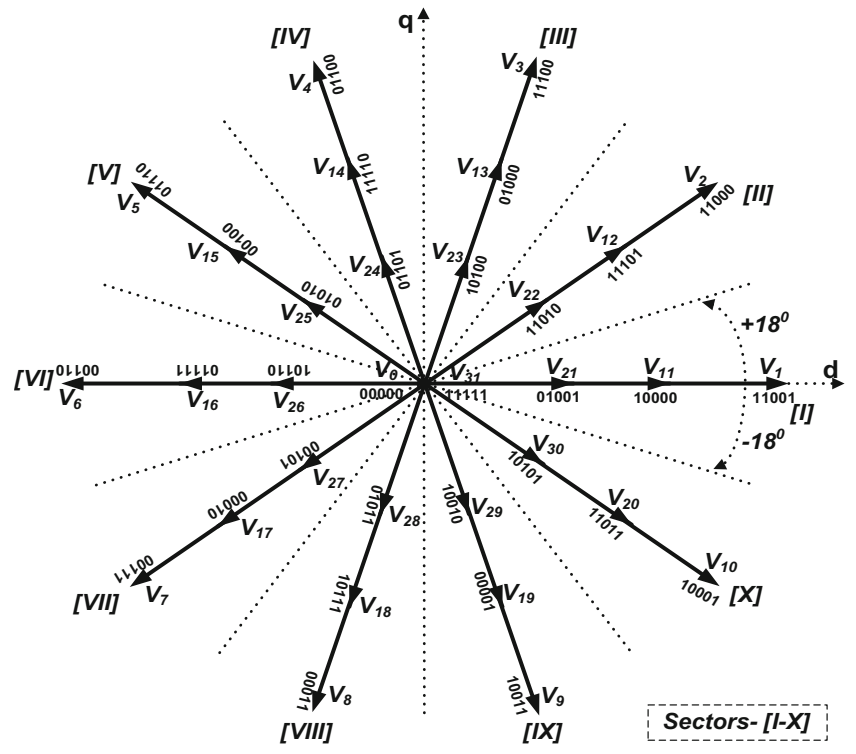

(a)

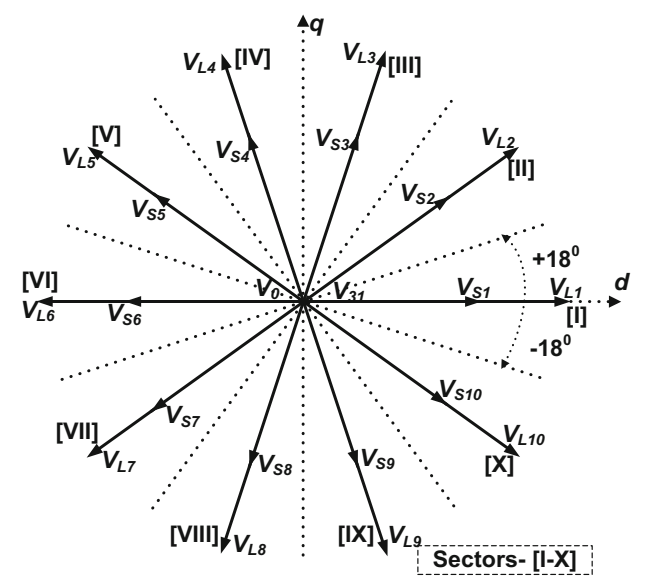

(c)

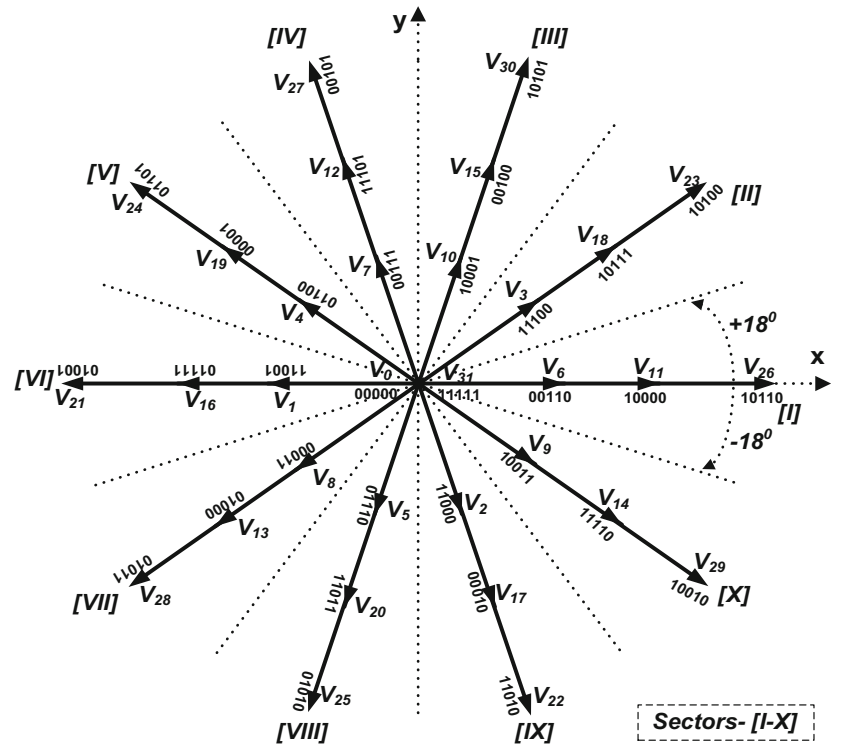

(b)

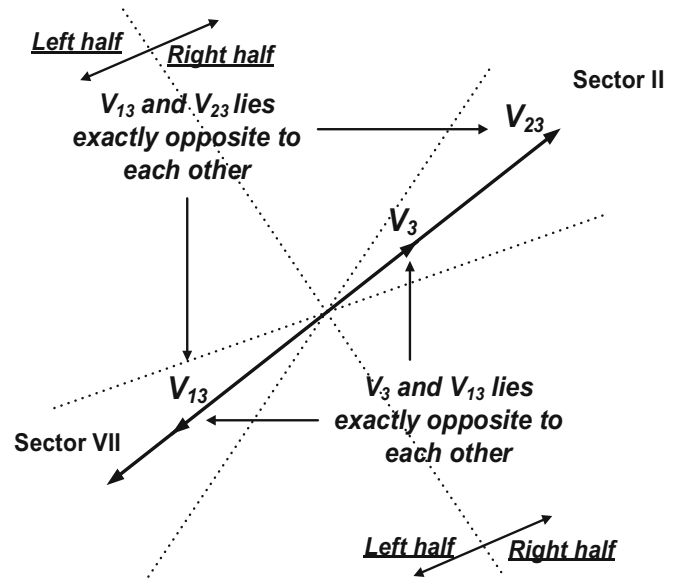

(d)

Figure 2. Mapping of the voltage vectors: (a) $d-q$ space plane, (b) $x-y$ space plane, (c) mapping of virtual voltage vectors in $d-q$ space plane and (d) oppositely located voltage vectors.

$$
(0.4+0.2472) V_{d c} / 2=0.3236 V_{d c} .
$$

The reason behind taking the average of dc-bus length of large and medium voltage vectors in case of large virtual voltage vectors and medium and small in case of small virtual voltage vectors is that both the types of actual voltage vectors in each virtual voltage vector group are employed for equal duration. From Eqs. (10)-(12), the comparison between the absolute values of torque variation due to virtual large voltage vectors $\left(\delta V_{L X}\right)$ and virtual small voltage vectors $\left(\delta V_{S X}\right)$ for incrementing the torque is

$$
\delta V_{L X}>\delta V_{S X}
$$

The comparison between the absolute values of torque variation due to virtual large voltage vectors, virtual small voltage vectors and zero voltage vectors $\left(\delta V_{0}\right)$ for decrementing the torque is

$$
\delta V_{L X}>\delta V_{S X}>\delta V_{0} .
$$

Both the types of virtual voltage vectors are responsible for incrementing as well as decrementing the torque, whereas zero voltage vectors are responsible for only decrementing the torque due to their zero dc-link utilization.

\subsection{Design of the proposed five-level torque comparator}

In the classical five-level torque comparator, the zero voltage vectors are used in the inner bands on both sides of 


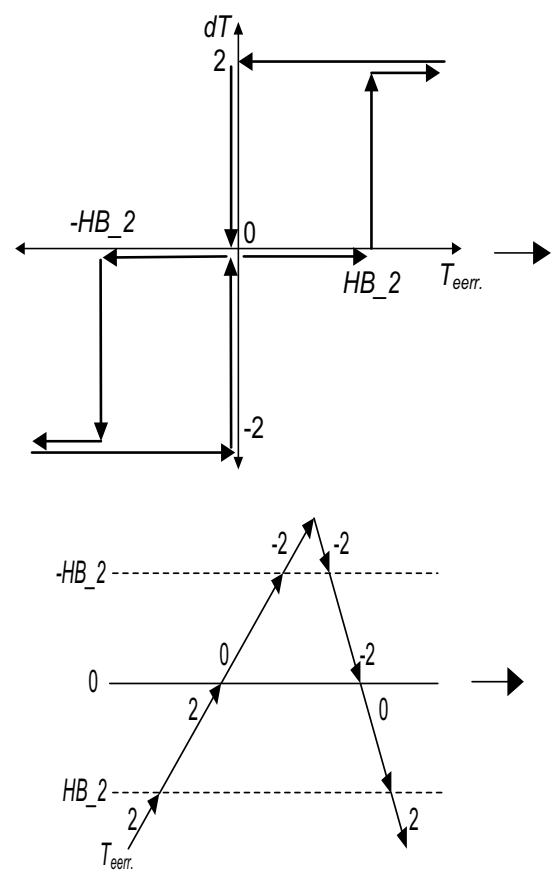

(a)
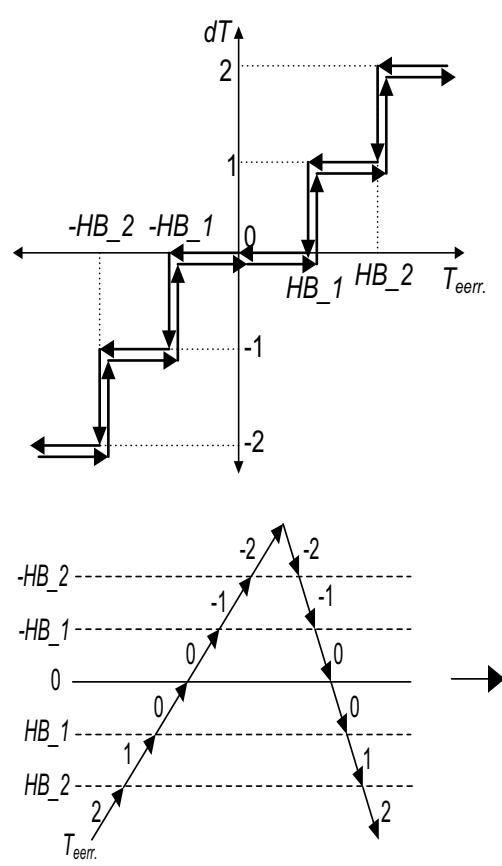

(b)
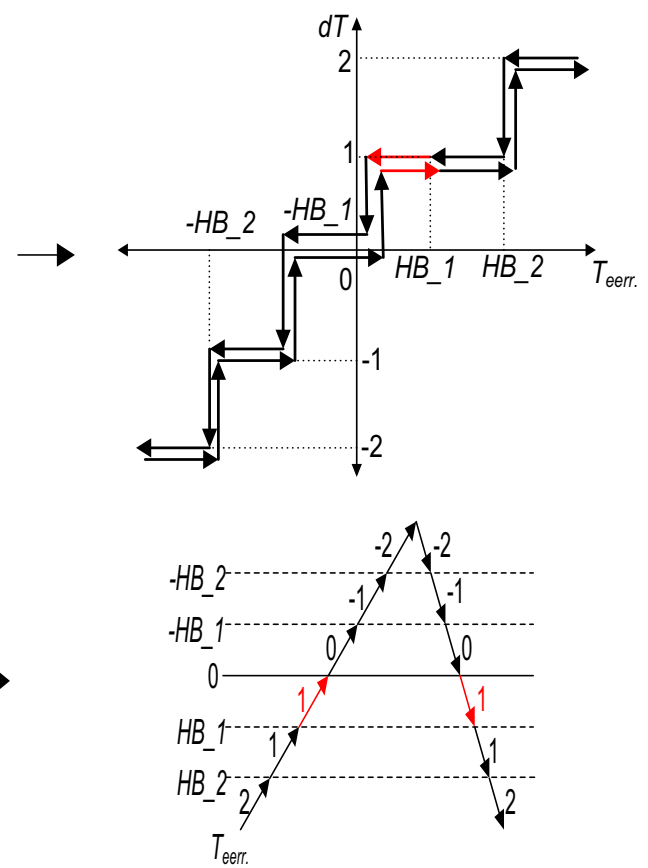

(c)

Figure 3. Representation of torque comparator: (a) classical three-level, (b) classical five-level and (c) proposed five-level torque comparator.

the comparator. If the zero voltage vectors are employed several times, the dc-link utilization will be affected. The small virtual voltage vectors can be employed instead of zero voltage vectors. This modification serves both the objectives, i.e., 1) torque ripple reduction and 2) improvement in dc-link utilization. Therefore, in order to gain these objectives, there are two corrections that need to be incorporated in the classical five-level torque comparator.

(a) When the torque error comes in the positive inner band from the positive end of the classical five-level torque comparator, the zero voltage vector $(d T=0)$ is selected as shown in figure $3 \mathrm{~b}$. Due to selection of zero voltage vector, the torque error does not reach its zero value; rather, it moves again towards positive end (torque error increases), because the actual torque is decreased. If zero voltage vector in the positive inner band is not replaced by small virtual voltage vector, the actual torque will never reach its reference value. If zero voltage vectors in the positive inner band are replaced by the small virtual voltage vectors $(d T=1)$, the actual torque will surely reach its reference value. Due to this, the torque error gets reduced and hence the torque ripple will be reduced. This modification is done in the proposed five-level torque comparator as shown in figure $3 \mathrm{c}$.

(b) When the torque error progresses from negative end, crosses its zero position and comes into the positive inner band of the classical five-level torque comparator, again zero voltage vector is selected as shown in figure $3 \mathrm{~b}$.
After crossing the zero position by the torque error, the torque error needs to be reduced (actual torque needs to be increased) immediately in order to reduce the torque ripple. However, selection of zero voltage vector here again decreases the torque, which enables the torque ripple to increase. The torque can be increased when the zero voltage vector is replaced by small virtual voltage vector $(d T=1)$ in the positive inner band. By selecting the small virtual voltage vectors the torque error gets reduced and hence the torque ripple gets reduced. This modification is done in the proposed five-level torque comparator as shown in figure $3 \mathrm{c}$.

There are three rules that need to be followed while designing the proposed five-level torque comparator.

1) The small virtual voltage vectors should be employed as many times as possible, but only within the outer bands [14]

The function of small virtual voltage vectors is to reduce the torque ripple. In the classical five-level torque comparator, the small virtual voltage vectors are utilized four times while incrementing and decrementing the torque error as shown in figure $3 \mathrm{~b}$. In case of proposed five-level torque comparator, number of times the small virtual voltage vectors are utilized is increased from four to six as shown in figure $3 \mathrm{c}$. It is observed in figure $3 \mathrm{c}$ that all the six small virtual voltage vectors are employed in the proposed comparator inside the outer band on both sides. 


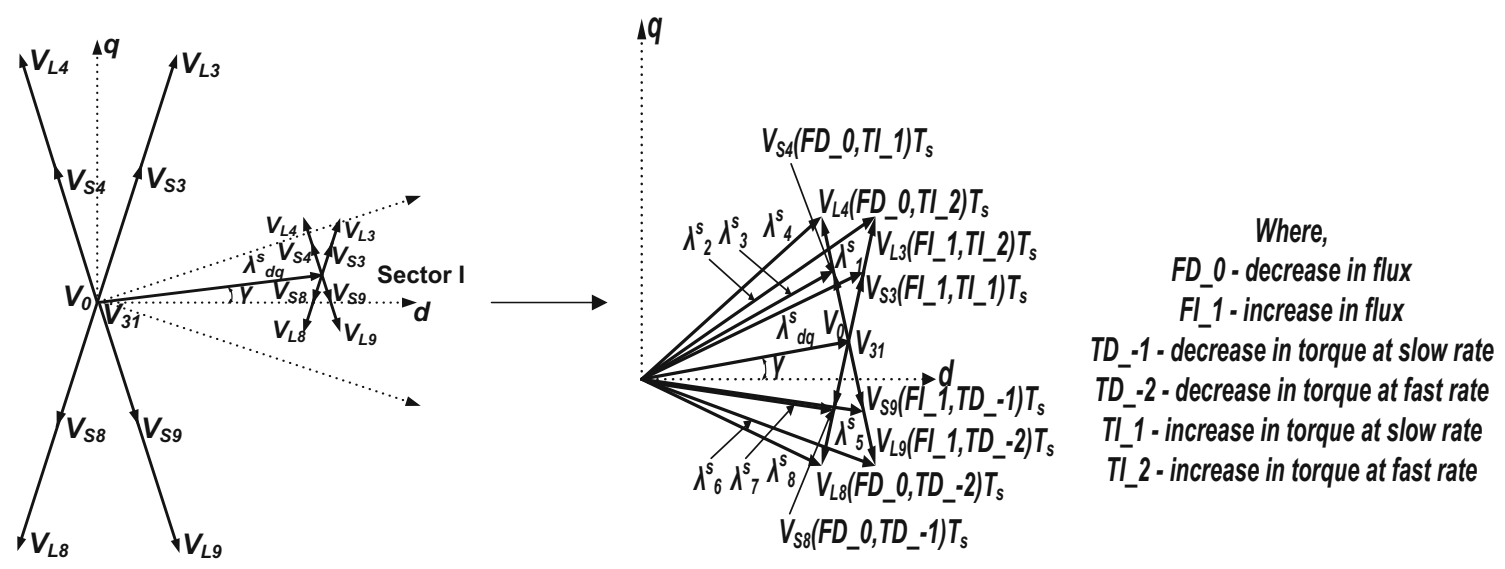

Figure 4. Selection of virtual voltage vectors for all the possible torque and flux comparator outputs for sector I.

Table 1. Virtual voltage vectors selection table.

\begin{tabular}{|c|c|c|c|c|c|c|c|c|c|c|c|}
\hline \multirow[b]{2}{*}{$d \lambda$} & \multirow[b]{2}{*}{$d T$} & \multicolumn{10}{|c|}{ Sector } \\
\hline & & I & II & III & IV & V & VI & VII & VIII & IX & $X$ \\
\hline \multirow[t]{5}{*}{1} & 2 & $V_{L 3}$ & $V_{L 4}$ & $V_{L 5}$ & $V_{L 6}$ & $V_{L 7}$ & $V_{L 8}$ & $V_{L 9}$ & $V_{L 10}$ & $V_{L 1}$ & $V_{L 2}$ \\
\hline & 1 & $V_{S 3}$ & $V_{S 4}$ & $V_{I S 5}$ & $V_{S 6}$ & $V_{S 7}$ & $V_{S 8}$ & $V_{S 9}$ & $V_{S 10}$ & $V_{S 1}$ & $V_{S 2}$ \\
\hline & 0 & $V_{0}$ & $V_{31}$ & $V_{0}$ & $V_{31}$ & $V_{0}$ & $V_{31}$ & $V_{0}$ & $V_{31}$ & $V_{0}$ & $V_{31}$ \\
\hline & -1 & $V_{S 9}$ & $V_{S 10}$ & $V_{S 1}$ & $V_{S 2}$ & $V_{S 3}$ & $V_{S 4}$ & $V_{S 5}$ & $V_{S 6}$ & $V_{S 7}$ & $V_{S 8}$ \\
\hline & -2 & $V_{L 9}$ & $V_{L 10}$ & $V_{L 1}$ & $V_{L 2}$ & $V_{L 3}$ & $V_{L 4}$ & $V_{L 5}$ & $V_{L 6}$ & $V_{L 7}$ & $V_{L 8}$ \\
\hline \multirow[t]{5}{*}{0} & 2 & $V_{L 4}$ & $V_{L 5}$ & $V_{L 6}$ & $V_{L 7}$ & $V_{L 8}$ & $V_{L 9}$ & $V_{L 10}$ & $V_{L 1}$ & $V_{L 2}$ & $V_{L 3}$ \\
\hline & 1 & $V_{S 4}$ & $V_{S 5}$ & $V_{S 6}$ & $V_{S 7}$ & $V_{S 8}$ & $V_{S 9}$ & $V_{S 10}$ & $V_{S 1}$ & $V_{S 2}$ & $V_{S 3}$ \\
\hline & 0 & $V_{31}$ & $V_{0}$ & $V_{31}$ & $V_{0}$ & $V_{31}$ & $V_{0}$ & $V_{31}$ & $V_{0}$ & $V_{31}$ & $V_{0}$ \\
\hline & -1 & $V_{S 8}$ & $V_{S 9}$ & $V_{S 10}$ & $V_{S 1}$ & $V_{S 2}$ & $V_{S 3}$ & $V_{S 4}$ & $V_{S 5}$ & $V_{S 6}$ & $V_{S 7}$ \\
\hline & -2 & $V_{L 8}$ & $V_{L 9}$ & $V_{L 10}$ & $V_{L 1}$ & $V_{L 2}$ & $V_{L 3}$ & $V_{L 4}$ & $V_{L 5}$ & $V_{L 6}$ & $V_{L 7}$ \\
\hline
\end{tabular}

2) The zero voltage vectors should be used inside the inner band on negative side of the comparator only

In the classical five-level torque comparator the zero voltage vectors are employed in the inner bands on both sides of the torque comparator as shown in figure $3 \mathrm{~b}$, whereas in the proposed five-level torque comparator, the zero voltage vectors are used inside the inner band on the negative side only as shown in figure $3 \mathrm{c}$, which justifies that the zero voltage vectors should be employed for decrementing the torque only. Since the zero voltage vectors have zero dc-bus length, it obviously affects the dc-link utilization. The proposed five-level torque comparator is designed keeping in mind that the maximum possible dc-link utilization should be obtained while reducing the torque ripple. Therefore, the zero voltage vectors are employed in proposed five-level comparator only where they are required (inside the inner band on the negative side of the comparator only).

3) The large virtual voltage vectors should be used outside the outer band on both sides of the comparator Equations (13) and (14) show that the torque variation due to utilization of large virtual voltage vectors is more than the torque variation due to utilization of small virtual voltage vectors. Therefore, the large virtual voltage vectors are employed outside the outer band on both sides of the comparator to fasten the torque response while incrementing or decrementing the actual torque.

\subsection{Selection of virtual voltage vectors through proposed five-level torque comparator}

The virtual voltage vectors are selected based on the information of the torque, $d-q$ stator flux and its location for increasing or decreasing the flux $(F I$ and $F D)$ and the torque $(T I$ and $T D$ ) as shown in figure 4 . This is further illustrated in much detail for sector I. For example, when the stator flux enters the first sector and when increase of the flux $\left(F I \_1\right)$ and increase of the torque (TI_2) are required, the virtual voltage vector $V_{L 3}$ is selected for a very short time $T_{s}$ (sampling time). The selection of virtual voltage vector $V_{L 3}$ increases the stator flux, because the resultant stator flux $\lambda_{2}^{s}$ is greater than the previous stator flux $\lambda_{d q}^{s}$ advances the stator flux in forward 
direction (assume that anticlockwise is the forward direction). Similarly, other virtual voltage vectors will be selected. The selection of voltage vectors in all the sectors for all the possible conditions of torque and flux comparator is provided in table 1 .

\subsection{Selection of actual voltage vectors}

Table 2 selects the actual voltage vector based on the output from table 1 and the location of $x-y$ stator flux. For example, if $V_{L 3}$ is selected by table 1 and if $x-y$ stator flux lies in third sector, the actual medium voltage vector $V_{13}$ is selected by table 2 . The voltage vector $V_{13}$ is selected when the $x-y$ stator flux lies in sector X-IV (X-I-II-III-IV), because the voltage vector $V_{13}$ lies exactly opposite to the $x-y$ stator flux location X-IV (X-I-II-IIIIV). For opposite half, i.e., when the $x-y$ stator flux lies in sector V-IX (V-VI-VII-VIII-IX), the large voltage vector $V_{3}$ is selected, because the voltage vector $V_{3}$ lies exactly opposite to the $x-y$ stator flux location V-IX (V-VI-VII-VIII-IX). Similarly, remaining actual voltage vectors will be selected. The selection of opposite voltage vectors to the location of $x-y$ stator flux does not allow the $x-y$ stator flux to increase. Therefore, by selecting the opposite voltage vectors to the $x-y$ stator flux, the magnitude of $x-y$ stator current can be controlled, which reduces the distortion in the $d-q$ stator current waveform. Once the virtual voltage vector is selected by table 1 , table 2 sees the location of $x-y$ stator flux $(\theta)$ and accordingly selects the actual voltage vector. The control block diagram of the proposed DTC method is shown in figure 5 , which mainly consists of proposed five-level torque comparator, classical two-level flux comparator, virtual voltage vector selection table 1 and actual voltage vector selection table 2 , which initiates the switching action in the inverter for satisfying the required condition of torque while maintaining the stator flux constant.

\section{Simulation results}

The operation of five-phase IM controlled by the DTC scheme with the classical and proposed five-level torque comparator is carried out through MATLAB simulation. The sampling frequency used for the simulation is $20 \mathrm{kHz}$. The motor has the rated torque of $6 \mathrm{Nm}$ and the rated flux of $0.54 \mathrm{~Wb}$. The outer and inner bands on both sides of both the classical and proposed five-level torque hysteresis controller are set, respectively, to $\pm 6.66 \%$ and $\pm 3.33 \%$ of the rated torque. For the two-level flux hysteresis controller, the bands are set to $\pm 0.55 \%$ of the rated flux. The data and parameters of the machine are depicted in table 3.

Figures 6 and 7 summarize the performance of fivephase IM controlled by DTC method with the classical fivelevel torque comparator [12] and the proposed five-level torque comparator, respectively. For the operation, the motor speed is fixed to $1000 \mathrm{rpm}$. It is seen that the proposed torque comparator has significantly reduced the

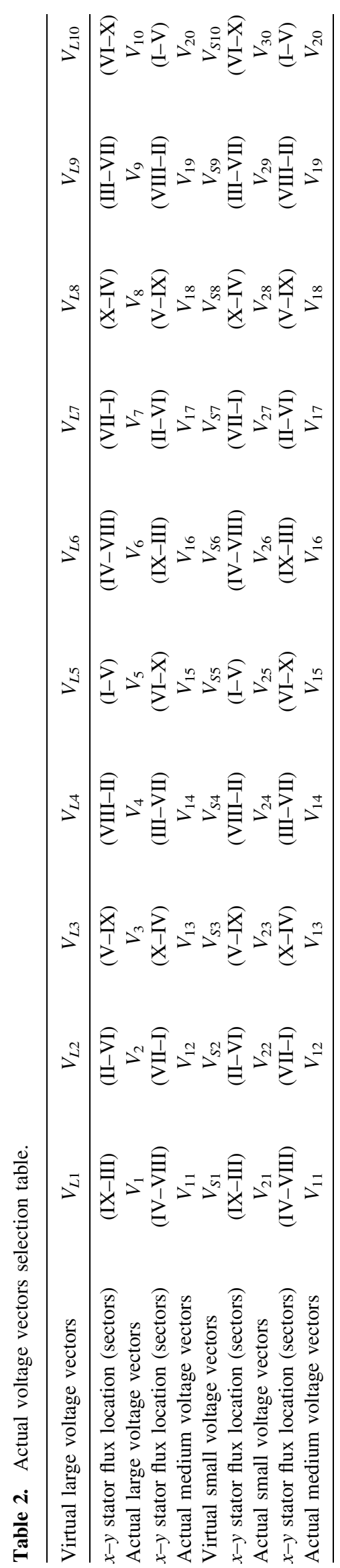




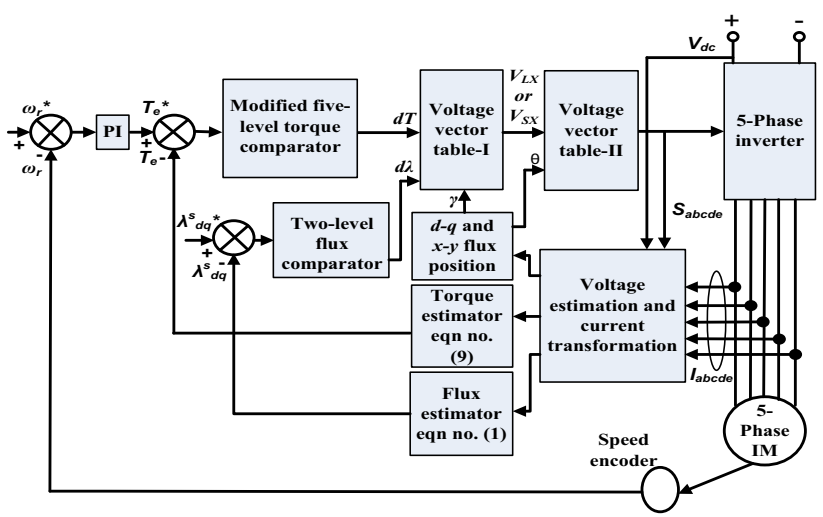

Figure 5. Control block diagram for the proposed DTC method.

Table 3. Induction motor data and parameters.

\begin{tabular}{lc}
\hline $5 \mathrm{ph}, 1 \mathrm{Hp}, 200 \mathrm{~V}, 50 \mathrm{~Hz}, 1400 \mathrm{rpm}$ \\
\hline$r_{s}$ & $0.8 \Omega$ \\
$r_{r}$ & $0.6 \Omega$ \\
$L_{l s}$ & $2.6 \mathrm{mH}$ \\
$L_{l r}$ & $2.6 \mathrm{mH}$ \\
$L_{m}$ & $151 \mathrm{mH}$ \\
Rotor inertia $J$ & $0.047 \mathrm{~kg} \mathrm{~m}^{2}$ \\
$P$ & 2 \\
\hline
\end{tabular}

torque ripple. The torque ripple in both the cases are shown to illustrate the torque ripple reduction. In both the cases, the stator flux of rated value is maintained constant throughout the operation. The no-load-current waveform is improved in case of the proposed DTC method. The virtual voltage vectors are implemented in both the cases. Therefore, the trajectory of $x-y$ stator current has reduced magnitude.

\section{Experimental results}

Figure 8 shows a block diagram of experimental set-up, which consists of five-phase IM as a test rig, DSP, an IGBT five-phase inverter, a signal conditioning circuit consisting of five current sensors out of which sufficient four are used, speed encoder and a dc-link voltage sensor. The control scheme is based on floating-point unit (FPU) $150 \mathrm{MHz}$ TMS320F28335 DSP board. This DSP offers controllability, reliability, programmability and flexibility to realize the digital control of the DTC scheme. To write C-codes, five ADC channels (four for current sensors and one for dc-bus voltage sensor) for signal conditioning circuit, one EPWM for starting of conversion from analogue to digital and five GPIOs for generating high or low gating signals were used. The sampling frequency of the control scheme is set to 20 $\mathrm{kHz}$. The average switching frequency of the inverter is around $3.2 \mathrm{kHz}$. The hysteresis bands in torque and flux

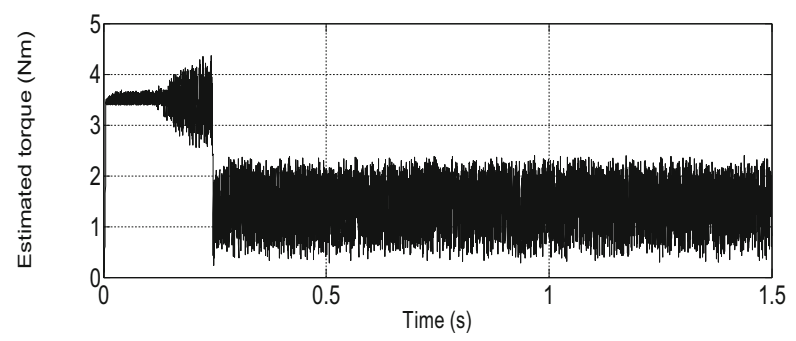

(a)

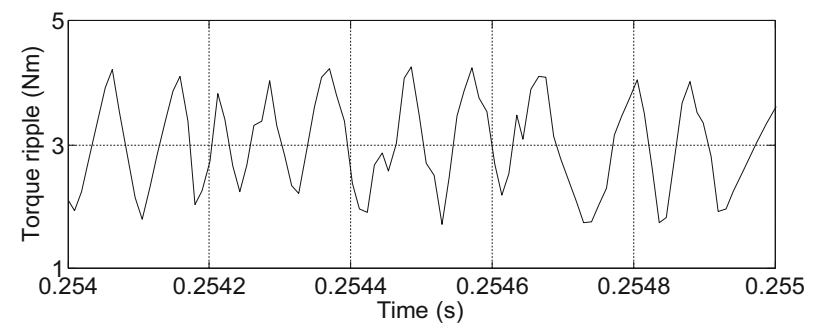

(b)

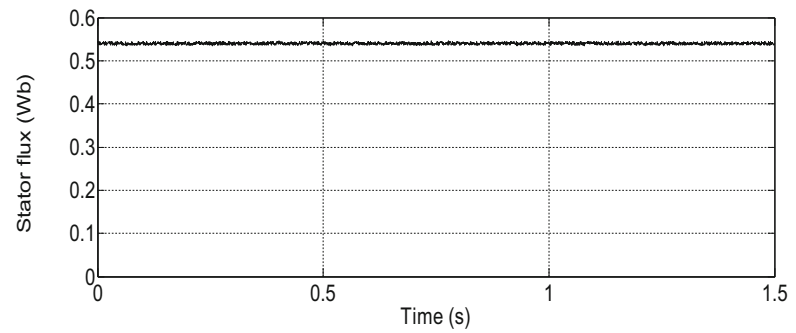

(c)

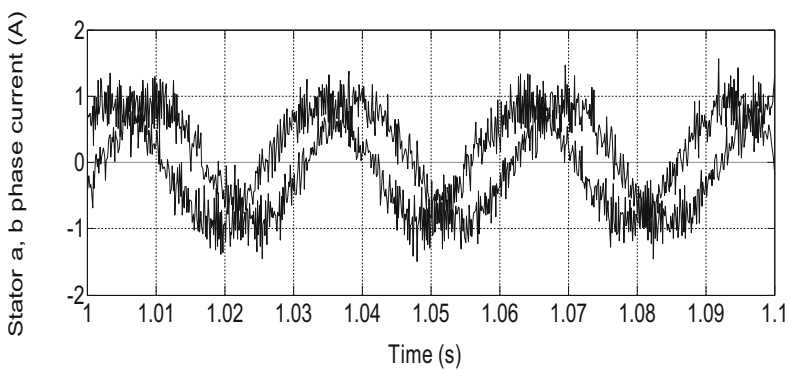

(d)

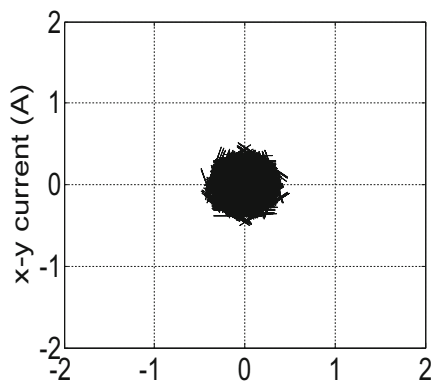

(e)

Figure 6. Simulation results of five-phase DTC with classical torque comparator: (a) torque, (b) toque ripple, (c) stator flux magnitude, (d) stator a, b phase current and (e) trajectory of $x-y$ stator current. 


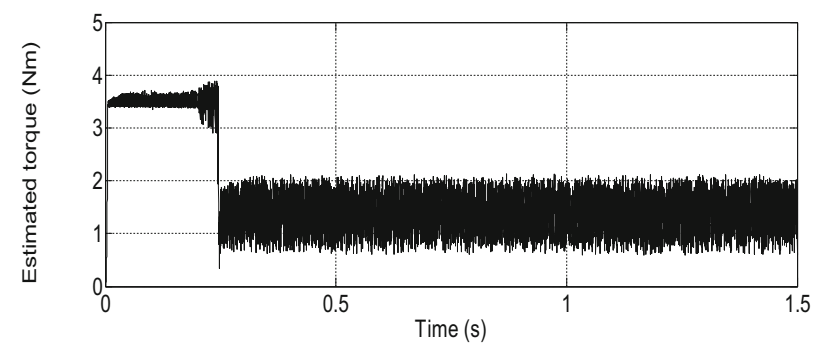

(a)

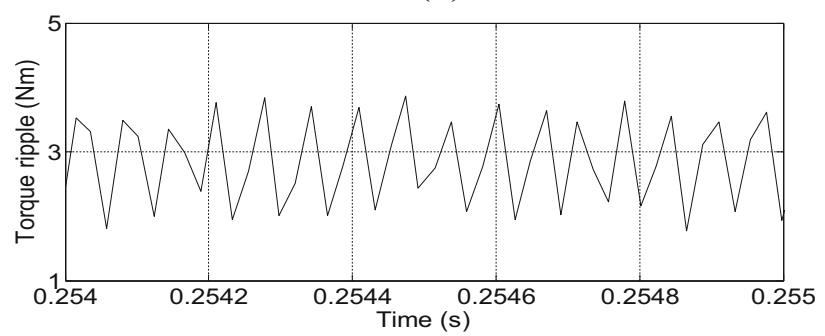

(b)

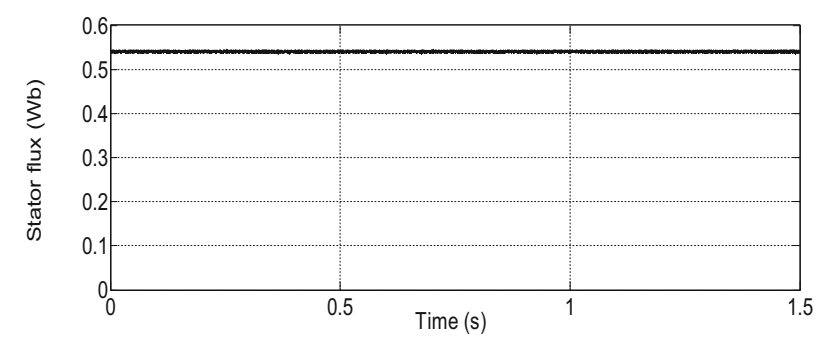

(c)
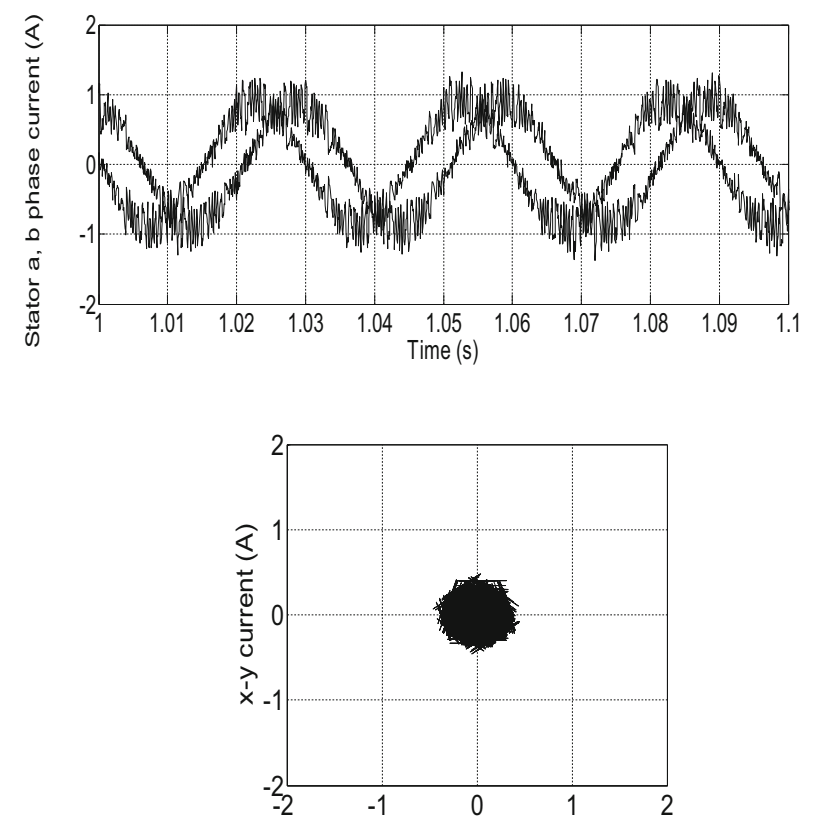

(e)

Figure 7. Simulation results of five-phase DTC with proposed torque comparator: (a) torque, (b) torque ripple, (c) stator flux magnitude, (d) stator a, b phase current and (e) trajectory of $x-y$ stator current. controllers are the same as in the simulation. The motor data and motor parameters are the same as those given in table 3.

Figures 9 and 10 summarize the experimental results of DTC schemes with the classical torque comparator and with the proposed torque comparator, respectively. The motor speed is fixed to $1000 \mathrm{rpm}$ throughout the operation. Since the torque sensors are not used due to their very high cost, the estimated torque is obtained from DSP through JTAG emulator. It is observed that by implementing the classical five-level torque comparator, the DTC scheme suffers from the torque ripple. The proposed torque comparator modifies the classical torque comparator and its effect can be observed in the form of torque ripple reduction as shown in figure 10a compared with figure 9a. The stator flux of the rated value is maintained constant throughout the operation in both DTC schemes as shown in figures $9 \mathrm{~b}$ and $10 \mathrm{~b}$. With the proposed comparator, the trajectory of $d-q$ stator current is better controlled, especially during steady-state operation (inner dense circle) as shown in figure 10c compared with trajectory of $d-q$ stator current as shown in figure $9 \mathrm{c}$. It is seen from the trajectories of $x-y$ stator current as shown in figures $9 \mathrm{c}$ and $10 \mathrm{c}$ that the magnitude of $x-y$ stator current is reduced, which proves the effective implementation of $x-y$ stator flux elimination algorithm in both cases.

Figures 11 and 12 summarize the experimental results of DTC schemes for the loading operation under the normal speed condition of $1000 \mathrm{rpm}$ with the classical torque comparator and with the proposed torque comparator, respectively. A load of $2.5 \mathrm{Nm}$ is applied to the motor. It is seen that the proposed comparator has effectively reduced the torque ripple during loading operation. It is observed that the current THD is reduced in the proposed DTC method. Therefore, the waveform of phase current is significantly improved. Since the same two-level flux comparator is implemented in both DTC schemes, the stator flux trajectory exhibits the same performance. It is inevitable that the stator flux in the $x-y$ space plane needs to be eliminated; otherwise the phase current will be distorted. Therefore, the virtual voltage vectors formation technique [12] has been implemented in both schemes to eliminate the $x-y$ stator flux. It is clearly seen that virtual voltage vectors formation technique did its function by almost eliminating the $x-y$ stator flux. Figures 13 and 14 show the steady-state and transient operation for classical and proposed comparator DTC methods. For the steady-state operation, the motor reference speed is fixed to $300 \mathrm{rpm}$ (almost $20 \%$ of the rated speed). It is seen that the proposed DTC scheme considerably did its work by reducing the torque ripple. Along with steady-state operation, transient operation is also carried out. Under this operation, a load of $3 \mathrm{Nm}$ is applied to the motor. As soon as the load is applied, the reference torque suddenly increases to the value set by the PI controller. The actual torque needs to immediately track the reference value in order to have the rapid dynamic 
Five-phase induction motor

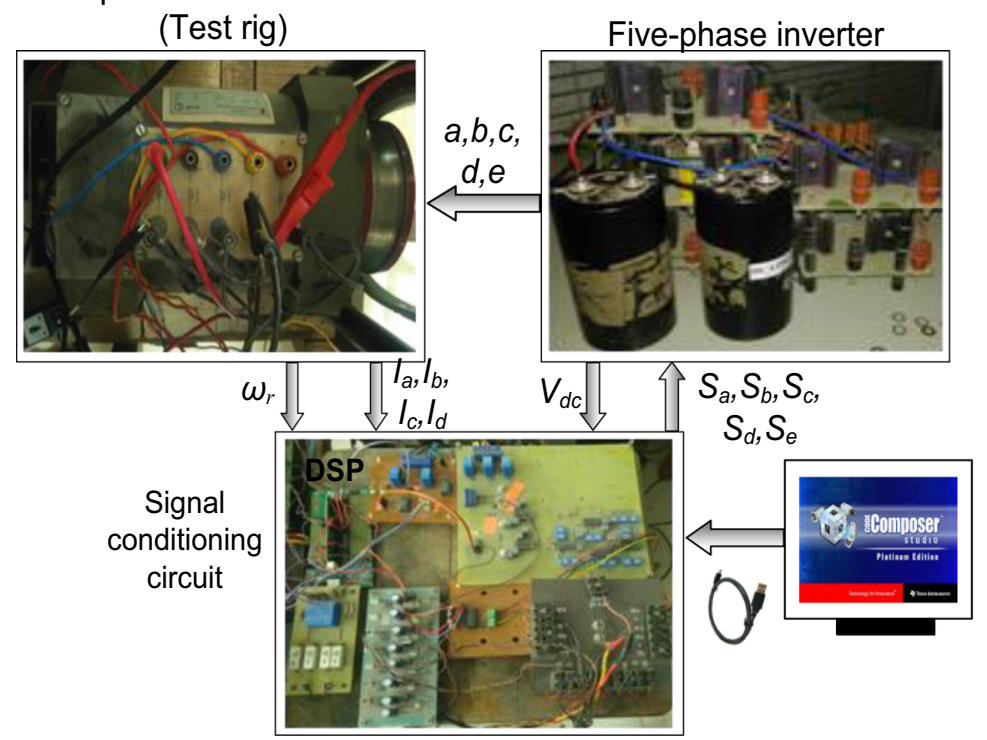

Figure 8. Block diagram of experimental set-up of five-phase inverter-fed DTC-controlled five-phase induction motor drive.

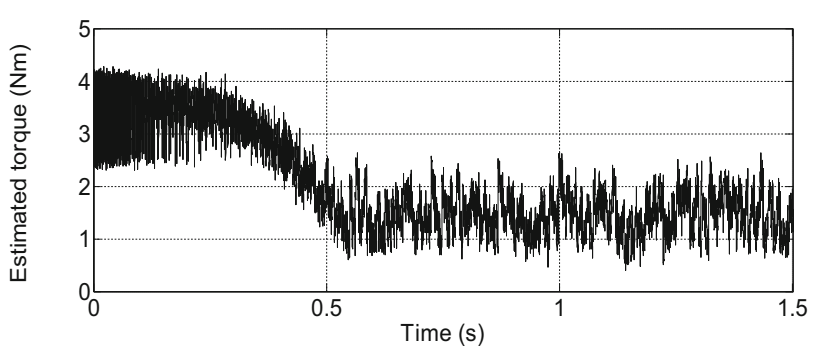

(a)

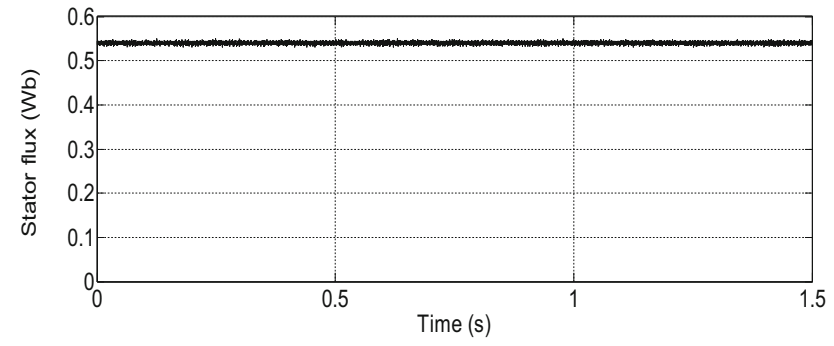

(b)
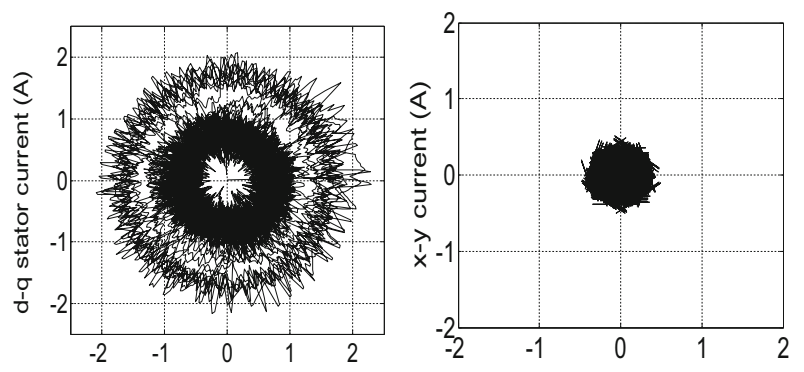

(c)

Figure 9. Experimental results of no-load operation of fivephase DTC with classical torque comparator: (a) torque, (b) stator flux and (c) trajectory of $d-q$ and $x-y$ stator current.

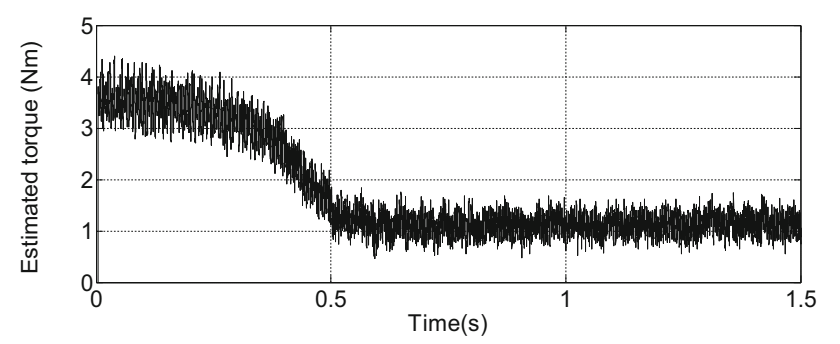

(a)

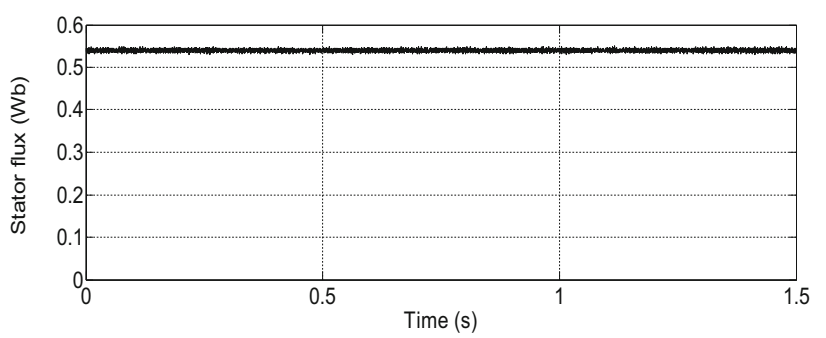

(b)
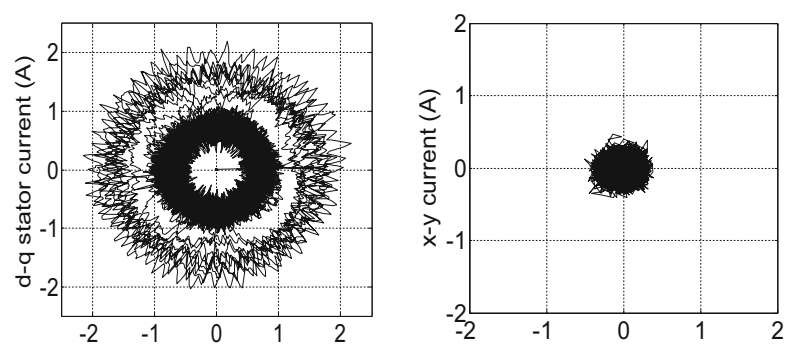

(c)

Figure 10. Experimental results of no-load operation of fivephase DTC with proposed torque comparator: (a) torque, (b) stator flux and (c) trajectory of $d-q$ and $x-y$ stator current. 


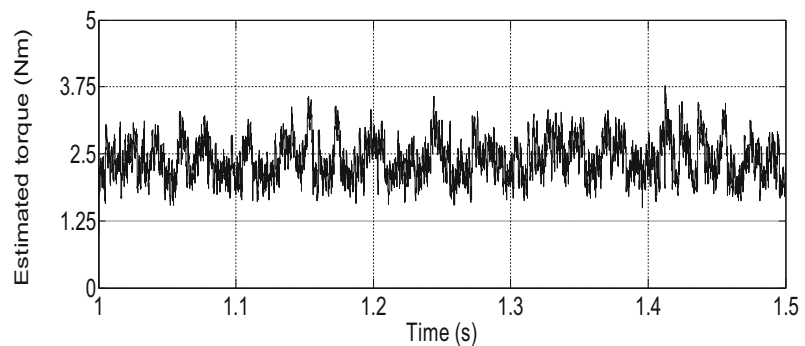

(a)

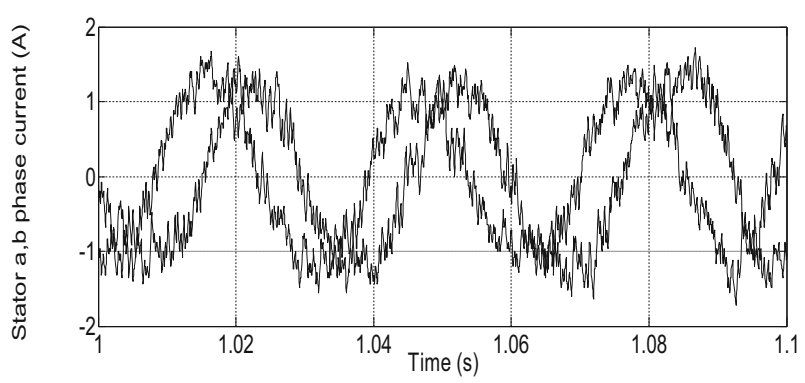

(b)

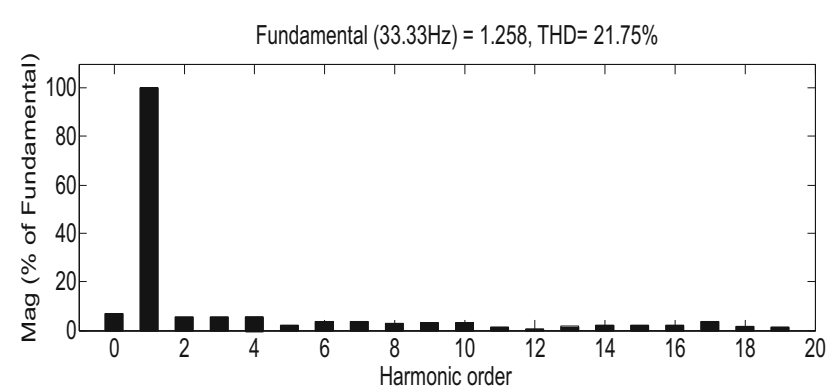

(c)
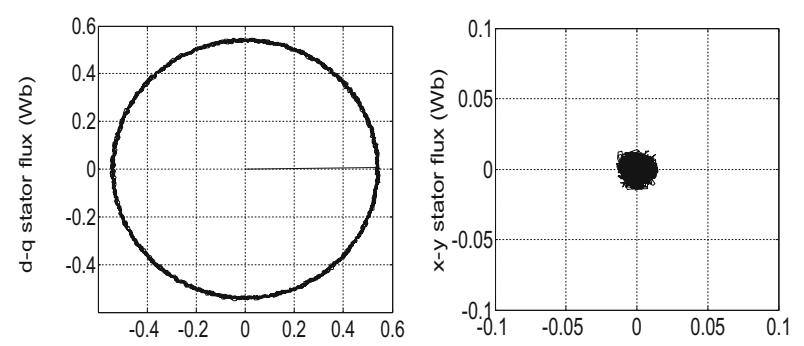

(d)

Figure 11. Experimental results of loaded steady-state operation of five-phase DTC with classical torque comparator: (a) torque, (b) stator a, b phase current, (c) current THD and (d) trajectory of $d-q$ and $x-y$ stator flux.

response. Since the large virtual voltage vector varies the torque faster as compared with small virtual voltage vector and zero voltage vector, large virtual voltage vector is applied in both DTC schemes during the transient period. Under transient operation, the speed reversal test is also conducted. When the speed reversal command is given, suddenly the PI controller generates negative torque command and hence the actual torque tries to follow the negative torque reference.

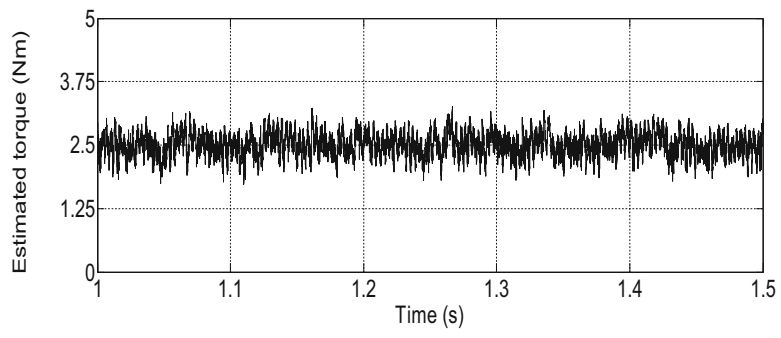

(a)

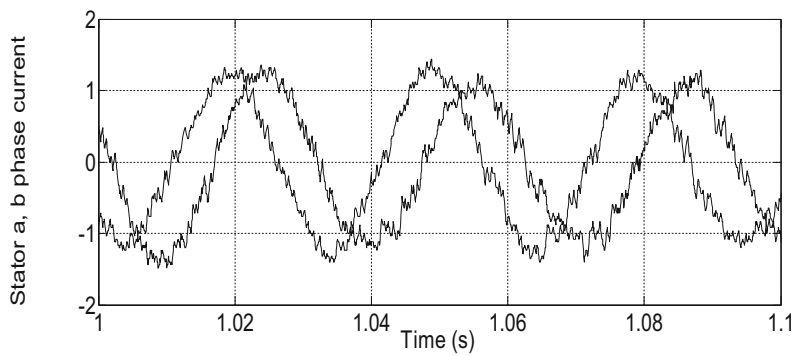

(b)

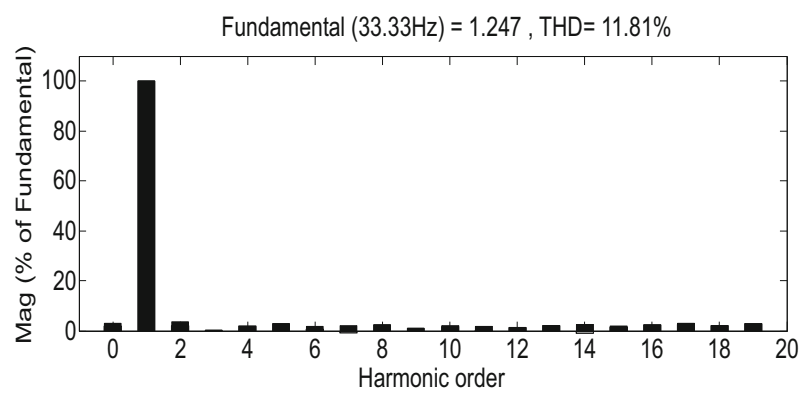

(c)
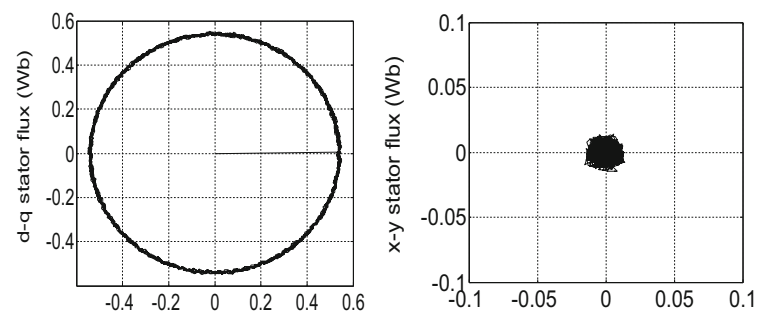

(d)

Figure 12. Experimental results of loaded steady-state operation of five-phase DTC with proposed torque comparator: (a) torque, (b) stator a, b phase current, (c) current THD and (d) trajectory of $d-q$ and $x-y$ stator flux.

Table 4 shows the comparison between the DTC schemes through experimental results during loading (2.5 $\mathrm{Nm}$ ) operation. It is observed that DTC with proposed fivelevel torque comparator has reduced the torque ripple by $38.89 \%$ compared with DTC with classical five-level torque comparator. It is seen that by implementing the proposed torque comparator, the dc-link utilization of the drive is improved by $7 \%$. It is also observed that with the proposed comparator the THD of current is significantly reduced. Figure 15 shows the average switching frequency under the 


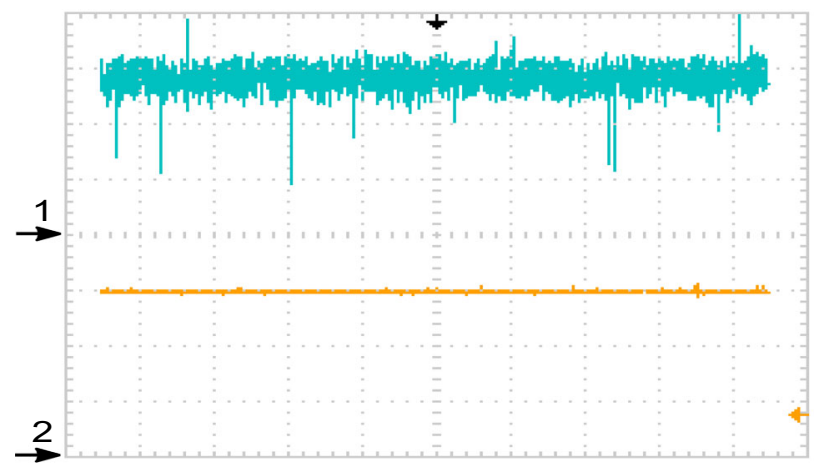

(I)

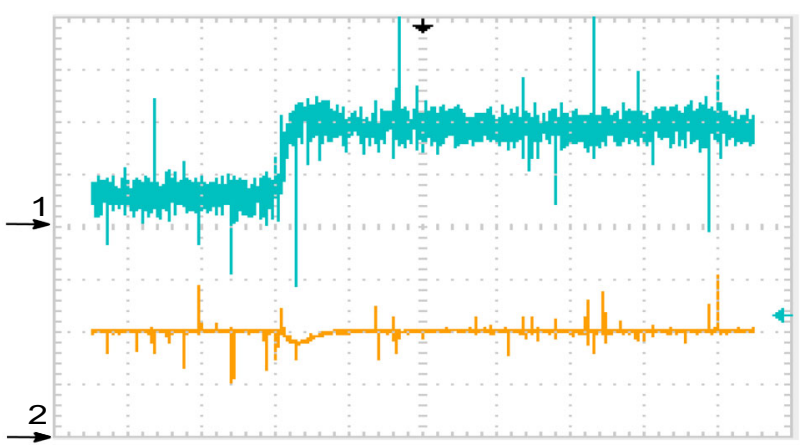

(II)

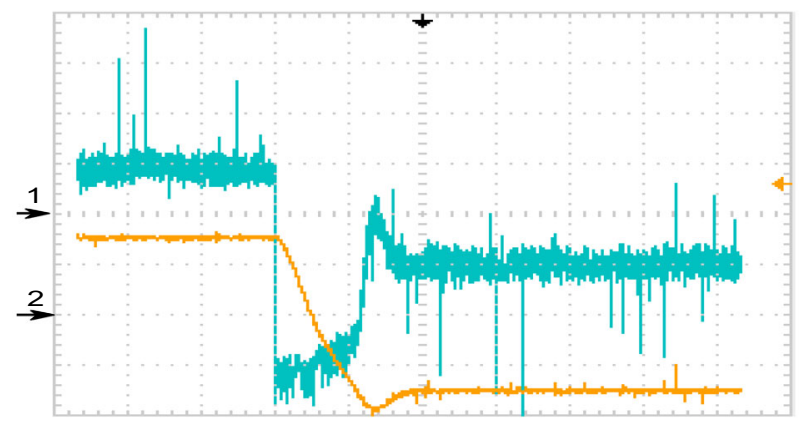

(III)

Figure 13. Experimental results for the classical comparator: (I) steady-state condition (CH. 1, torque $2 \mathrm{Nm} / \mathrm{div}$. and $0.5 \mathrm{~s} / \mathrm{div}$., CH.2 rotor speed $100 \mathrm{rpm} / \mathrm{div}$. and $0.5 \mathrm{~s} / \mathrm{div}$.), (II) transient condition of application of load $(\mathrm{CH}$. 1, torque $2 \mathrm{Nm} /$ div. and 0.5 s/div., CH.2 rotor speed $500 \mathrm{rpm} / \mathrm{div}$. and $0.5 \mathrm{~s} / \mathrm{div}$.) and (III) transient condition of speed reversal operation $(\mathrm{CH} .1$, torque 2 $\mathrm{Nm} /$ div. and $0.5 \mathrm{~s} / \mathrm{div}$., $\mathrm{CH} .2$ rotor speed $500 \mathrm{rpm} / \mathrm{div}$. and 0.5 s/div.).

operation of change in speed from 200 to $1400 \mathrm{rpm}$. It is seen that in both of the presented DTC schemes the average switching frequency remains almost the same. It is also observed that with increase in speed, the average switching frequency increases in both cases.

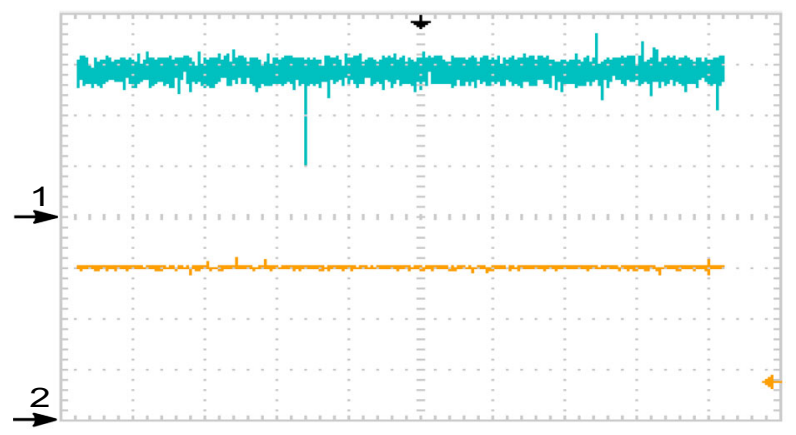

(I)

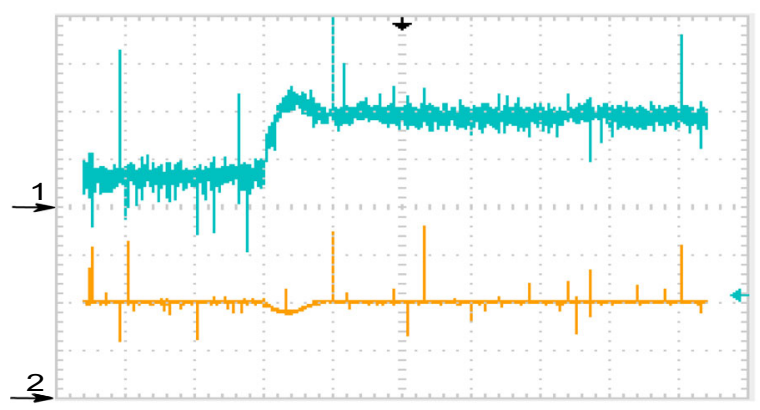

(II)

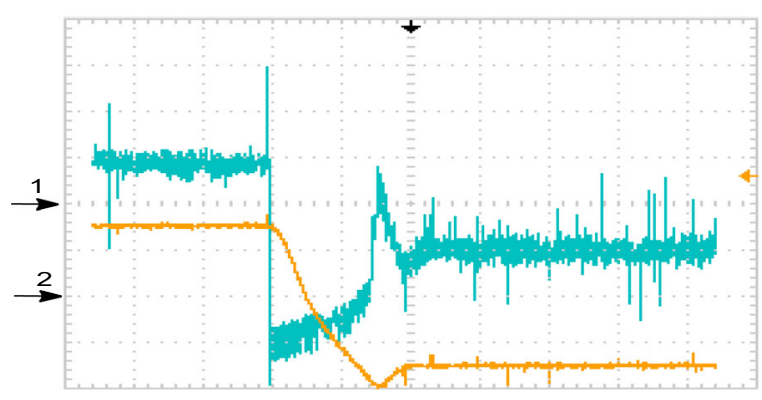

(III)

Figure 14. Experimental results for the proposed comparator: (I) steady-state condition (CH. 1, torque $2 \mathrm{Nm} / \mathrm{div}$. and $0.5 \mathrm{~s} / \mathrm{div}$., CH.2 rotor speed $100 \mathrm{rpm} / \mathrm{div}$. and $0.5 \mathrm{~s} / \mathrm{div}$.), (II) transient condition of application of load $(\mathrm{CH}$. 1, torque $2 \mathrm{Nm} /$ div. and 0.5 s/div., CH.2 rotor speed $500 \mathrm{rpm} / \mathrm{div}$. and $0.5 \mathrm{~s} / \mathrm{div}$.) and (III) transient condition of speed reversal operation $(\mathrm{CH}$. 1, torque 2 $\mathrm{Nm} /$ div. and $0.5 \mathrm{~s} / \mathrm{div}$., CH.2 rotor speed $500 \mathrm{rpm} / \mathrm{div}$. and 0.5 s/div.).

Table 4. Comparison of DTC schemes based on experimental data.

\begin{tabular}{lcccc}
\hline $\begin{array}{l}\text { DTC } \\
\text { schemes }\end{array}$ & $\begin{array}{c}\text { Peak-peak } \\
\text { torque } \\
\text { ripple }(\mathrm{Nm})\end{array}$ & $\begin{array}{c}\text { dc-link } \\
\text { utilization RMS } \\
\text { value of } V_{L L} / V_{d c}\end{array}$ & $\begin{array}{c}\text { Max. } x-y \\
\text { stator flux } \\
(\mathrm{Wb})\end{array}$ & $\begin{array}{c}\text { Current } \\
\text { THD } \\
(\%)\end{array}$ \\
\hline Classical & 1.8 & 0.58 & 0.016 & 21.63 \\
Proposed & $\underline{1.1}$ & $\underline{0.621}$ & $\underline{0.017}$ & $\underline{11.61}$ \\
\hline
\end{tabular}




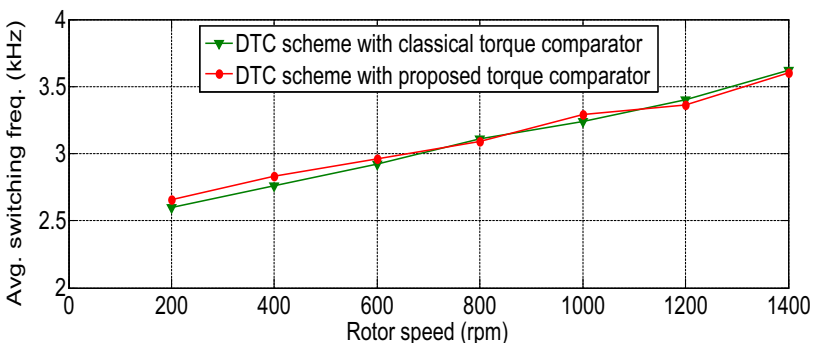

Figure 15. Experimental result of average switching frequency with respect to different speed conditions.

\section{Conclusion}

The paper proposed a modified five-level torque comparator against the classical five-level torque comparator in order to minimize the torque ripple and to improve the dclink utilization. The voltage vector selection through the classical five-level torque comparator affects the torque ripple and the dc-link utilization. The proposed torque comparator modifies the voltage vector selection inside the inner band on the positive side of the classical five-level torque comparator, which significantly reduces the torque ripple and improves the dc-link utilization. The $x-y$ stator flux magnitude has been eliminated in the proposed DTC scheme, which enables the stator phase current to follow the sinusoidal nature. The advantage of the DTC based on a hysteresis controller, i.e., the simplicity of the control algorithm, is retained through the proposed five-level torque comparator.

\section{Nomenclature}

DTC direct torque control

$\lambda_{d q}^{s} \quad d-q$ stator flux

$\lambda_{d q}^{r} \quad d-q$ rotor flux

$\lambda_{x y}^{s} \quad x-y$ stator flux

$\lambda_{x y}^{r} \quad x-y$ rotor flux

$V_{d q}^{s} \quad d-q$ stator voltage vector

$V_{d q}^{r} \quad d-q$ rotor voltage vector

$V_{x y}^{s} \quad x-y$ stator voltage vector

$V_{x y}^{r} \quad x-y$ rotor voltage vector

$i_{d q}^{s} \quad d-q$ stator current vector

$i_{d q}^{r} \quad d-q$ rotor current vector

$i_{x y}^{s} \quad x-y$ stator current vector

$i_{x y}^{r} \quad x-y$ rotor current vector

$r_{s} \quad$ stator resistance

$r_{r} \quad$ rotor resistance

$L_{l s} \quad$ leakage stator inductance

$L_{l r} \quad$ leakage rotor inductance

$L_{m} \quad$ mutual inductance

$P \quad$ pole pairs

$L_{s} \quad$ stator inductance

$L_{r} \quad$ rotor inductance $\sigma \quad$ leakage coefficient

$V_{L X} \quad$ large virtual voltage vectors

$V_{S X} \quad$ small virtual voltage vectors

$\frac{d T_{e}}{d t} \quad$ torque variation

$\delta V_{L X} \quad$ absolute value of torque variation due to virtual large voltage vectors

$\delta V_{S X}$ absolute value of torque variation due to virtual small voltage vectors

$\delta V_{0} \quad$ absolute value of torque variation due to zero voltage vectors

$T_{e 0} \quad$ torque at current instant

$\omega_{m} \quad$ motor angular velocity

\section{References}

[1] Pavithran K N, Parimelalagan R and Krishnamurthy M R 1988 Studies on inverter-fed five-phase induction motor drive. IEEE Trans. Power Electron. 3(2): 224-235

[2] Levi E 2008 Multiphase electric machines for variable-speed applications. IEEE Trans. Ind. Electron. 55(5): 1893-1909

[3] Levi E, Bojoi R, Profumo F, Toliyat H A and Williamson S 2007 Multiphase induction motor drives-a technology status review. Electr. Power Appl. IET 1(4): 489-516

[4] Takahashi I and Noguchi T 1986 A new quick response and high efficiency control strategy of an induction motor. IEEE Trans. Ind. Appl. 22(5): 820-827

[5] Xu H, Toliyat $\mathrm{H}$ and Petersen L 2002 Five-phase induction motor drives with DSP-based control system. IEEE Trans. Power Electron. 17(4): 524-533

[6] Casadei D and Serra G 1997 Analytical investigation of torque and flux ripple in DTC schemes for induction motors. In: Proceedings of IEEE IECON'97, pp. 552-556

[7] Kang J K and Sul S K 1999 New direct torque control of induction motor for minimum torque ripple and constant switching frequency. IEEE Trans. Ind. Appl. 35(5): 1076-1082

[8] Shyu K K, Lin J K, Van Pham V T, Yang M J and Wang T W 2010 Global minimum torque ripple design for direct torque control of induction motor drives. IEEE Trans. Ind. Electron. 57(9): 3148-3155

[9] Lee K B, Song J H, Choy I and Yoo J Y 2002 Torque ripple reduction in DTC of induction motor driven by three-level inverter with low switching frequency. IEEE Trans. Power Electron. 17(2): 255-263

[10] Zheng B L, Fletcher J E and He X 2011 A novel direct torque control scheme for a sensorless five-phase induction motor drive. IEEE Trans. Ind. Electron. 58(2): 503-513

[11] Riveros J, Barrero F, Levi E, Duran M, Toral S and Jones M 2013 Variable-speed five-phase induction motor drive based on predictive torque control. IEEE Trans. Ind. Electron. 60(8): 2957-2968

[12] Gao L, Fletcher J and Zheng L 2011 Low-speed control improvements for a two-level five-phase inverter-fed induction machine using classic direct torque control. IEEE Trans. Ind. Electron. 58(7): 2744-2754

[13] Iqbal A, Ahmed S M, Khan M A, Khan M R and Abu-Rub H 2010 Modeling, simulation and implementation of a five- 
phase induction motor drive system. In: Proceedings of Power Electronics, Drives and Energy Systems (PEDES) and 2010 Power India, 2010 Joint International Conference, pp. 1-6

[14] Ren Y and Zhu Z Q 2015 Reduction of both harmonic current and torque ripple for dual three-phase permanent- magnet synchronous machine using modified switchingtable-based direct torque control. IEEE Trans. Ind. Electron. 62(11): 6671-6683 